\title{
Guidelines for the Efficient Design of Sinewave Generators for Analog/Mixed-Signal BIST
}

\author{
Manuel J. Barragán, Diego Vázquez, Adoración Rueda, and José Luis Huertas \\ Instituto de Microelectrónica de Sevilla/Centro Nacional de Microelectrónica/ \\ Consejo Superior de Investigaciones Científicas (IMSE-CNM-CSIC)/Universidad de Sevilla \\ Av. Americo Vespucio s/n, 41092, Sevilla, Spain. \\ E-mail: manuelj@imse.cnm.es
}

\begin{abstract}
$\bar{\equiv}$
Abstract- This paper presents a design methodology for the implementation of efficient and accurate sinewave generators suitable for analog and mixed-signal BIST applications. The design guidelines are based on an analytical discussion that contemplates the main non-idealities of the generator. A full design example is presented to illustrate the proposed methodology.
\end{abstract}

\section{INTRODUCTION}

The increasing complexity of current and future ICs has the associated issue of more complex, longer, and hence more expensive test. This issue is identified in the SIA Roadmap for Semiconductors [1] as one of the key problems for future mixed-signal SoCs.

The main test difficulties are due to the test of the analog parts. They are traditionally tested using costly functional approaches, but their sensitivity to loading effects, environmental conditions and process variations make their test a difficult task that usually demands expensive ATEs (Automatic Test Equipment).

Built-In Self-Test (BIST) techniques have been identified as a way to overcome some of the problems cited above. They consist of moving part of the required test resources (test stimuli generation, response evaluation, test control circuitry, etc.) into the chip [1].

Most of the mixed-signal subsystems in complex ICs (filters, ADCs, DACs, signal conditioners, etc.) can be characterized and tested (frequency domain specifications, linearity, etc.) using periodic signals (square, triangular, sinusoidal, etc.). The on-chip generation of this kind of stimuli is of main importance, and hence, some interesting work has paid attention to it [2]-[6].

In this line, this paper presents an efficient methodology for the design of the sinewave generator with reduced circuitry requirements reported by the authors in [6].

The paper is organized as follows. Section II reviews briefly the functionality of the generator. In Section III, the main non-idealities of the generator and their impact in the generator performance are discussed to define a design methodology. Section IV provides a full design example to

978-1-4244-7791-3/10/\$26.00 (C)2010 IEEE illustrate the proposed methodology. Finally, Section V remarks the key points of this work in the conclusions.

\section{REVIEW OF THE GENERATOR FUNCTIONALITY}

The sinewave generator in [6] consists of a modified filtering stage which includes the generation of the desired signal and the filtering of unwanted frequency components.

To explain the functionality of the generator, let us consider the two systems depicted in Fig.1. The system in Fig.1a is a standard filtering stage with transfer function $H(s)$, that is excited by an input signal $f(t)$. On the other hand, the system in Fig. $1 \mathrm{~b}$ is identical, but its input elements, that is, the elements that link the input signal with the core of the filter, vary in time according to function $f(t)$, and it is excited by a unit DC level. The work in [6] demonstrates that the response of both systems in the steady state is the same.

The system in Fig.1b, as a response to a DC input, provides an output signal that is the function $f(t)$ filtered accordingly to $H(s)$. It should be clear that if $f(t)$ is a periodic function, then the system will output also a periodic signal. If the transfer function $H(s)$ removes all the non-desired frequency components, this periodic output will become a sinusoidal-like waveform.

It is important to notice that the amplitude and frequency of the generated waveform can be programmed. The constant

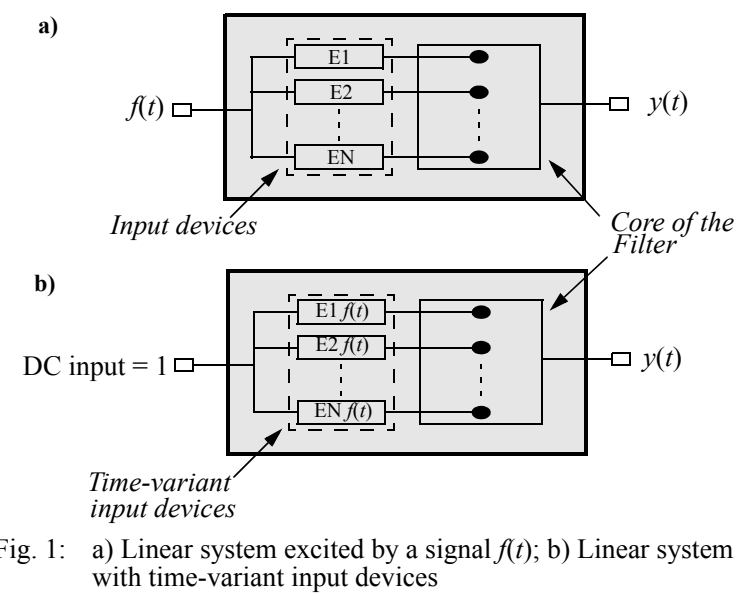


input of the system acts as a scaling factor that controls the amplitude of the generated signal, while its frequency can be controlled by function $f(t)$ and the tuning of the system transfer function.

Function $f(t)$ is related to the input devices of the considered system. Regarding to the generation of sinewave signals, the work in [6] demonstrates that a good candidate for $f(t)$ is a step-wise sinewave. This choice allows a simple implementation: step-wise variation of the input devices of a system can be achieved simply by switching devices. Regarding the system transfer function, what is needed is to match it to the shape and frequencies of interest in the application. In this sense, lowpass and bandpass sections are very convenient for this application, whenever the main harmonic of function $f(t)$ is in the passband and the non-desired components in the rejection band.

\section{LIMITING FACTORS AND DESIGN METHODOLOGY}

In order to find efficient design guidelines, it is important to consider the performance limitations of the proposed strategy that will have to be analyzed during the design stage. In this line, the main limitations of the proposed approach are given by:

- The realization of function $f(t)$ : restricting the discussion to the case of step-wise sinewaves, both the number of steps per period, $N$, and the possible errors affecting the step levels, $\varepsilon$, will have a direct impact on the performance.

- The selected linear system: the shape of its transfer function $H(s)$ defines the attenuation of the non-desired frequency componets of function $f(t)$, while its non-idealities in terms of non-linear behavior will introduce distortion in the output signal.

These limiting factors have to be considered in the design stage to implement a generator with a desired performance. In fact a trade-off can be defined between the number of step levels composing $f(t)$ and the selectivity and linearity of the filtering stage. Thus, a high number of levels in $f(t)$ will push its spectral replicas to high frequencies, which allows to relax the specifications of the filter, but increases the overhead and complexity in the implementation of $f(t)$. On the contrary, a low number of step levels reduces this complexity, but the spectral replicas are in this case closer to the main frequency and will need a sharper filter to be attenuated. In addition, the errors in the step levels together with the non-linearity of the filtering stage will introduce harmonic distortion in the output signal.

The study of these limitations can be synthesized in an efficient design methodology. Fig.2 shows the proposed design flow for the generator, from the definition of the goal

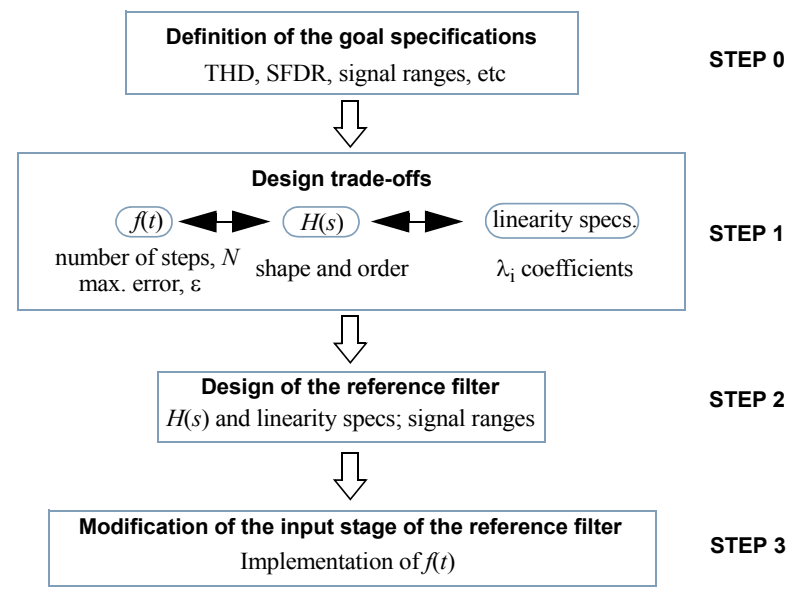

Fig. 2: Conceptual design flow for the proposed generator

specifications to its final design. Let us describe each step in the proposed design methodology.

\section{A. Step 1: design trade-offs}

Firstly, the trade-off between the choice of $f(t), H(s)$, and the system non-linearity has to be considered to get a set of parameters compatible with the desired generator performance defined in the Step 0.

The analysis of this trade-off can be performed using a high-level model, that is shown in Fig.3. This model contemplates the main limitations of the generator. It has three main blocks: an input block which generates the function $f(t)$, an ideal linear filter with transfer function $H(s)$, and a non-linear block which models the non-linearity of the system.

Concerning $f(t)$, the design variables that have to be found in this step are the number of levels and its maximum tolerable error. The first one is a design choice, while the second one is related to mismatching and process variations, and hence, is determined by the selected technology.

Regarding the linear system, in this design step the transfer function $H(s)$ has to be chosen. What is needed is to keep the main tone of $f(t)$ in the passband of the filter, and attenuate its unwanted frequency components. The linearity of the system depends strongly on the particular filtering stage, however, in this design stage it can be modelled by a polynomial in the form,

$$
y(t) \approx x(t)+\lambda_{2} x^{2}(t)+\lambda_{3} x^{3}(t)+\ldots
$$

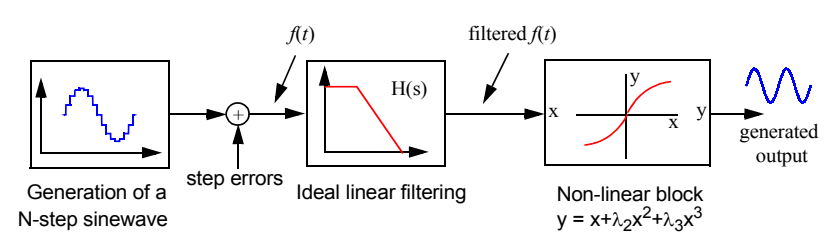

Fig. 3: High level model of the proposed sinewave generator 
where $x(t)$ represents the output of the ideal linear filtering, coefficients $\lambda_{i}$ model the non-linearity of the system, and $y(t)$ is the output of the system. Usually, a third-order polynomial is enough for modeling purposes. Coefficients $\lambda_{i}$ obtained with this high level model will be mapped to circuit parameters once the filter architecture is selected.

\section{B. Step 2: Design of the reference filter}

Once that the transfer function of the filter, $H(s)$, and its non-linearity behavior (in terms of the $\lambda_{i}$ coefficients) have been obtained, in this design step the designer has to select a particular filter architecture. For this choice the signal ranges defined in the Step 0, in terms of amplitude and frequency, have to be considered. For instance, high-frequency operation can be reached using OTA-C architectures, while low-frequency and wide amplitude range should require a switched-capacitor (SC) architecture. In this stage, the $\lambda_{i}$ coefficients have to be mapped into lower level system specifications. For instance, in OTA-C filters there is a direct relation between these coefficients and the linearity of the transconductors.

\section{Step 3: Modification of the input stage of the filter}

Once that the filtering stage is defined, to complete the design of the generator the input stage of the filter has to be modified to implement the $f(t)$ function selected in the first step (see Fig.1b). The errors in the step levels have to be kept below the maximum error levels obtained in Step 1. These errors can be related to technological parameters. For instance, in a SC implementation they are related to the matching of the capacitors, while for OTA-C systems they depend on the matching between transconductors.

The modification of the input stage completes the design of the generator. In fact, the design flow is very similar to the design of an analog filter, except for the modification of the input stage.

\section{DESIGN EXAMPLE}

As an example of application, this section presents the design of a continuous-time sinewave generator in a $0.35 \mu \mathrm{m}$ 4-metal 2-poly 3.3V CMOS technology. The general set of goal specifications are listed in Table I.

Table I:Goal specifications for the OTA-C sinewave generator

\begin{tabular}{|c|c|}
\hline Parameter & Value \\
\hline \hline Frequency range & from $20 \mathrm{MHz}$ to $40 \mathrm{MHz}$ \\
\hline Amplitude range & up to $250 \mathrm{mV}$ peak-to-peak \\
\hline Linearity & $\begin{array}{c}\text { better than } 50 \mathrm{~dB} \text { THD and SFDR in the } \\
\text { desired frequency and amplitude range }\end{array}$ \\
\hline
\end{tabular}

\section{A. Step 1: design trade-offs}

The first step in the design of the generator is to define the characteristics of the function $f(t)$ and the filtering stage compatible with the desired specifications. Let us consider first the trade-off between the number of step-levels, its maximum error, and the transfer function $H(s)$. To reduce the design space, the search will be restricted to second-order low-pass filtering sections with transfer function,

$$
H(s)=\frac{k \omega_{0}^{2}}{s^{2}+\left(\omega_{0} / Q\right) s+\omega_{0}^{2}}
$$

where $k$ is a scaling factor, $Q$ is the quality factor of the complex conjugate poles, and $\omega_{0}$ is its frequency. This choice is motivated by the good results predicted in [6] for this family of filtering sections.

The frequencies of the unwanted harmonics of function $f(t)$ have to be placed in the rejection band of the system, while the desired tone (its main harmonic) is placed in the passband of the filter. In this particular case $f(t)$ is a $N$-step sinewave of period $N T_{S}\left(T_{s}\right.$ is the duration of each step), and $H(s)$ is described by (2), so the best choice is clearly to make,

$$
\omega_{0}=\frac{2 \pi}{N T_{s}}
$$

To estimate the effect of the ideal filtering, a Monte Carlo analysis using maximum errors of $0.1 \%$ and $1 \%$ in the levels of the stepwise sinewave and applied to the considered filtering section with different quality factors, $Q$, has been carried out.

a)

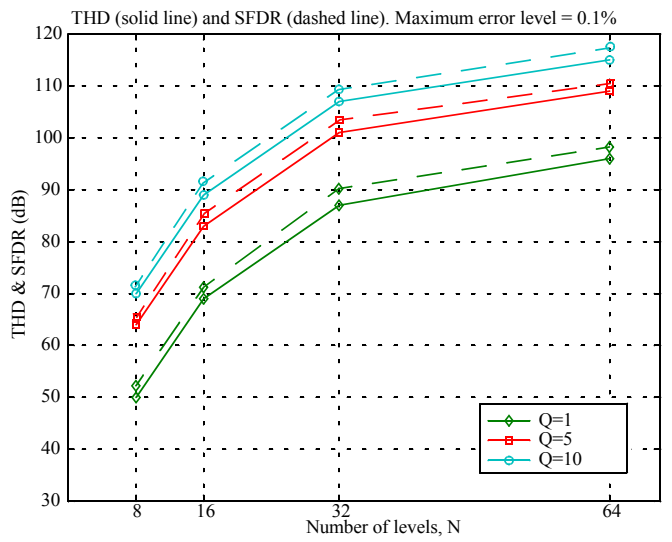

b)

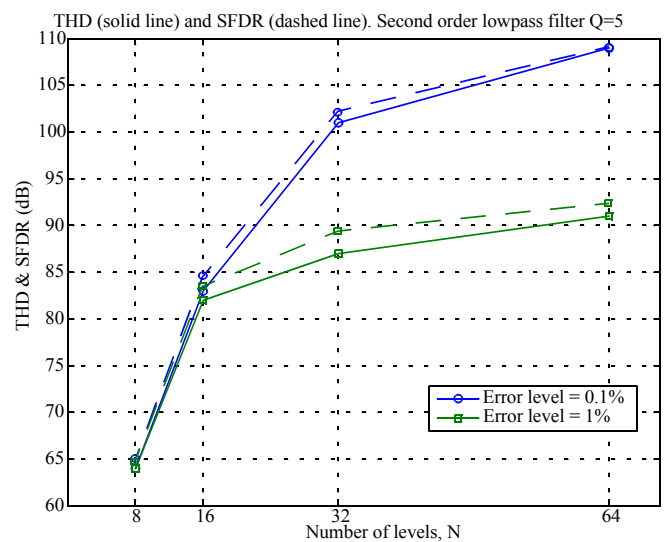

Fig. 4: Obtained THD and SFDR figures as a function of the number of step levels: a) for different $\mathrm{Q}$ factors; $b$ ) for different error levels 
Fig.4a shows the obtained worst cases in terms of SFDR and THD for a maximum error in the step levels of $0.1 \%$ as a function of the number of step levels and $Q$. On the other hand, Fig.4b shows the worst cases for $Q=5$ as a function of the number of step levels and its maximum error.

In the view of the results in Fig.4, the combination of a 16-step sinewave as $f(t)$ and a second-order lowpass filtering stage with a relatively low quality factor $Q$ around $Q=5$, gives THD and SFDR figures above the desired specifications (see Fig.4), while representing a good trade-off solution between performance and design complexity. It is interesting to notice that using only 8 step levels may be also a viable solution. However, our choice of 16 levels pushes the spectral replicas to higher frequencies and gives a conservative margin over the goal specifications in terms of linearity.

Concerning the linearity of the filtering stage, assuming the third-order polynomial model (1), Fig.5 shows an exploration of the design space in terms of the non-linear coefficients $\lambda_{2}$ and $\lambda_{3}$. In the view of this exploration, and assuming that coefficient $\lambda_{2}$ can be made close to zero using a fully-differential architecture, the third-order coefficient $\lambda_{3}$ has to be lower than $750 \mathrm{mV} / \mathrm{V}^{3}$ in the defined signal range in order to fulfill the desired linearity specifications in Table 3.1. Under these circumstances, the step-levels of function $f(t)$ can be affected by deviations up to $10 \%$ of its intended value without degrading the linearity of the generated signal. Although this deviation range may seem relatively high, it is coherent with the previous design choices: the linearity of the generator is mainly determined by the linearity of the filter core, and in a lesser extent by the first spectral replica, so the errors in the

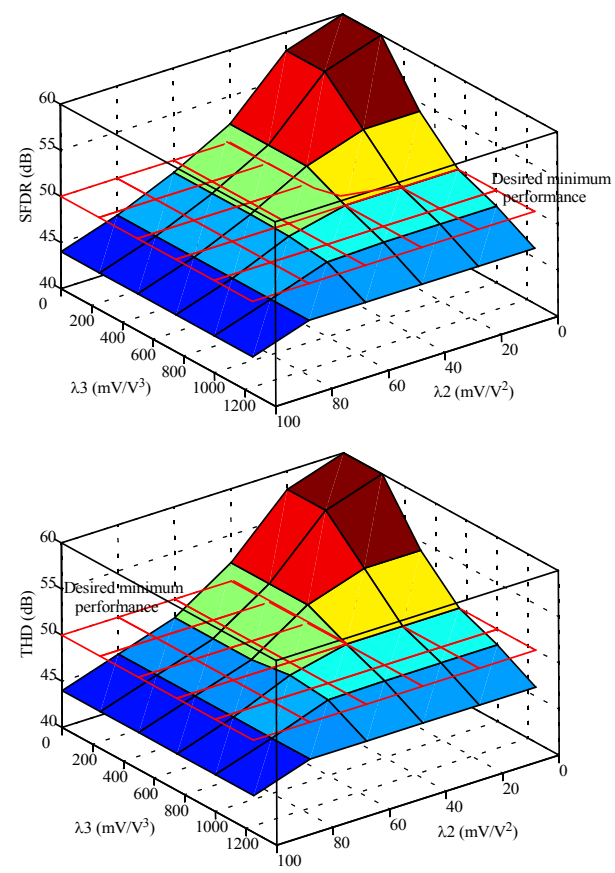

Fig. 5: SFDR and THD estimations using a third order model for a $250 \mathrm{mVpp}$ signal. The plane at $50 \mathrm{~dB}$ marks the desired minimum performance

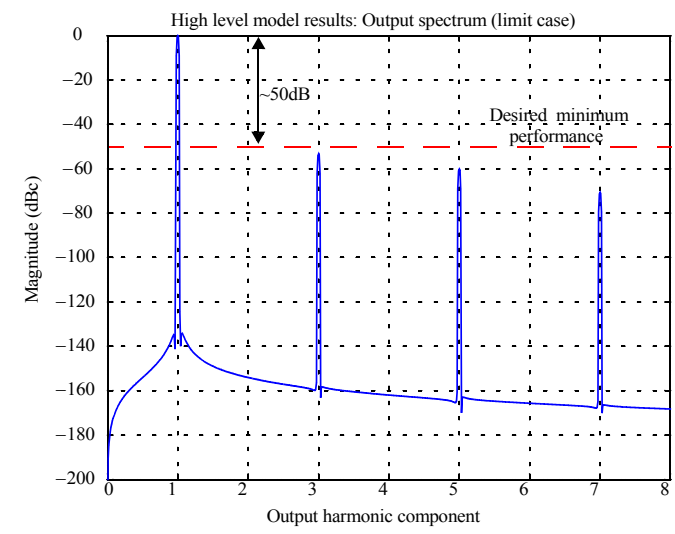

Fig. 6: Output spectrum obtained with the high level model for the limit case $\left(N=16\right.$, error level $=10 \%, \lambda_{2}=0, \lambda_{3}=750 \mathrm{mV} / \mathrm{V}^{3}$, output amplitude $=250 \mathrm{mVpp}$ )

step levels have little impact in the performance. Fig.6 shows the spectrum of the generator output obtained with the high level model under the discussed limit conditions of error levels and linearity. The obtained results meet the minimum goal performance.

\section{B. Step 2: Design of the reference filter}

The selected filter block diagram is shown in Fig.7. It is a typical fully-differential second-order lowpass OTA-C filter [7]. The choice of an OTA-C architecture has been motivated by the relatively high operation frequency defined in Table I. The transfer function of the system, $V_{\text {out }} / V_{\text {input }}$ in the Laplace domain can be easily obtained by simple analysis,

$$
H(s)=\frac{4\left(G_{m A} / C_{1}\right)\left(G_{m C} / C_{2}\right)}{s^{2}+s\left(\mathrm{G}_{m B} / C_{1}\right)+4\left(G_{m D} / C_{1}\right)\left(G_{m C} / C_{2}\right)}
$$

Taking into account the desired generator specifications, Table II shows a set of design parameters compatible with these choices. The transconductor values shown in Table II correspond to the needed transconductor tuning range to achieve the extreme frequency range values $20 \mathrm{MHz}$ and $40 \mathrm{MHz}$ approximately, a pole quality factor of 5 , and a scale factor $k=1$, which gives a peak gain $(\sim k Q)$ of around $14 \mathrm{~dB}$.

Table II:Design parameters in Fig.7 (tuning range for cut-off frequency from $20 \mathrm{MHz}$ to $40 \mathrm{MHz}$ )

\begin{tabular}{|cc|cc|}
\hline $\mathbf{G}_{\mathbf{m A}}$ & 275 to $550 \mu \mathrm{A} / \mathrm{V}$ & $\mathbf{G}_{\mathbf{m D}}$ & 275 to $550 \mu \mathrm{A} / \mathrm{V}$ \\
\hline $\mathbf{G}_{\mathbf{m B}}$ & 55 to $110 \mu \mathrm{A} / \mathrm{V}$ & $\mathbf{C}_{\mathbf{1}}$ & $4 \mathrm{pF}$ \\
\hline $\mathbf{G}_{\mathbf{m C}}$ & 275 to $550 \mu \mathrm{A} / \mathrm{V}$ & $\mathbf{C}_{\mathbf{2}}$ & $4 \mathrm{pF}$ \\
\hline
\end{tabular}

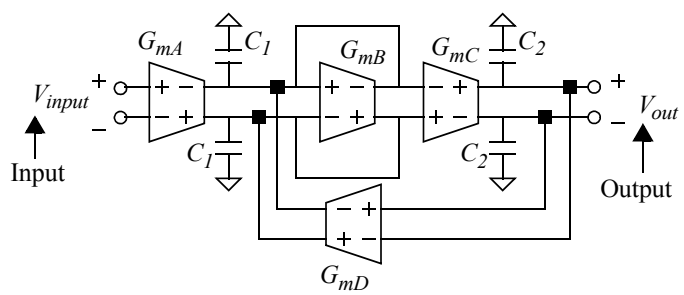

Fig. 7: Block diagram of the OTA-C reference filter 
The design parameters have been chosen using matching considerations. Thus, capacitors have been made equal, and transconductors are integer multiples of a unit transconductor. That is, transconductors $G_{m A}, G_{m C}$, and $G_{m D}$ have been chosen to be five times larger than the unit transconductor $G_{m B}$, and can be built from five matched unit transconductors $G_{m B}$ in parallel.

Concerning the linearity specifications, the non-linear characteristic of a fully-differential transconductor can be approximated by a third order polynomial in the form,

$$
I_{\text {out }} \approx G_{m} V_{\text {in }}+\alpha_{3} V_{\text {in }}^{3}
$$

where $I_{\text {out }}$ is the output current of the transconductor, $V_{\text {in }}$ is its input voltage, $G_{m}$ is its linear transconductance, and $\alpha_{3}$ is a third-order non-linear coefficient. To assure that the goal linearity specifications are fulfilled, high level simulations of the whole filter were performed sweeping the $\alpha_{3}$ coefficient. According to the obtained results, the non-linear coefficient $\alpha_{3}$ of the unit transconductor has to be kept below $50 \mu \mathrm{A} / \mathrm{V}^{3}$ in the selected signal range.

\section{Step 3: Modification of the input stage}

Following the proposed design methodology, the next step in the design flow is to modify the input stage of the developed OTA-C reference filter to transform it into the desired OTA-C sinewave generator. The block diagram of the developed generator is shown in Fig.8a. The only modification with respect to the reference filter is that the input transconductor $G_{m A}$ has been replaced by a switching scheme controlled by the digital signal $\Phi_{i n}$ and a programmable transconductor $G_{m a}(t)$ to implement the step-wise sinewave levels. Transconductor $G_{m a}(t)$ is composed by four transconductors in parallel $\left(G_{m 1}\right.$ to $\left.G_{m 4}\right)$ as depicted in Fig.8b, whose contributions are switched on or off in an incremental way accordingly to the time scheme shown in Fig.8c. This way, the required 5 steps of the positive half sinewave are generated, while the input switching scheme controlled by signal $\Phi_{i n}$ sets the weight, positive or negative, of the step. The resulting transconductance $G_{m a}(t)$ can be described as,

$$
G_{m a}(t)=\left\{\Phi_{i n}(t)-\bar{\Phi}_{i n}(t)\right\}\left\{\sum_{k=1}^{4} \Phi_{k}(t) G_{m k}\right\}
$$

where

$$
G_{m k}=G_{m A}\left[\sin \left(\frac{k \pi}{8}\right)-\sin \left(\frac{(k-1) \pi}{8}\right)\right]
$$

The possible errors in the step levels defined by $G_{m a}(t)$ are due to two main factors: the possible mismatching between transconductors, which may shift the transconductance of the steps, and the offset of each elemental transconductor $G_{m k}$, which adds to the output current of the programmable transconductor. These deviations depend on the selected elemental OTA architecture. Fig.9 shows the transistor level

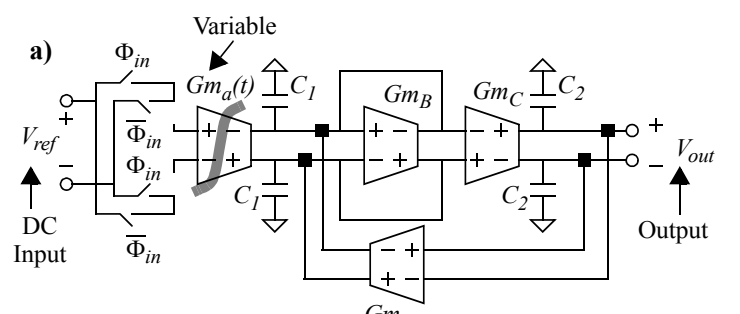

b)

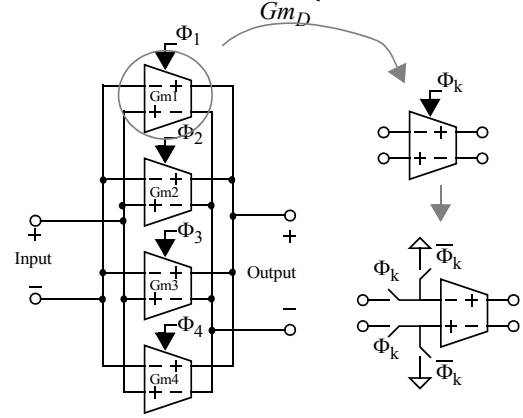

c)

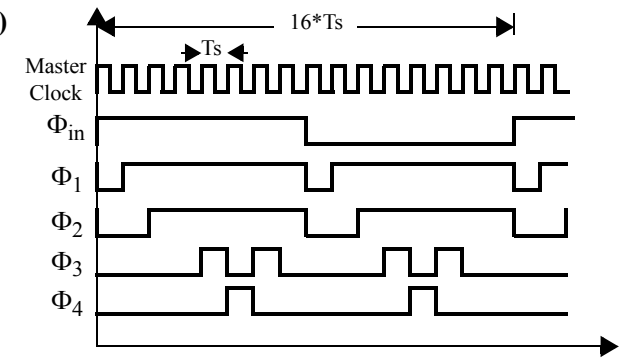

Fig. 8: a) Block diagram of the signal generator. b) Time variant transconductor implementation. c) Time scheme

schematic of the elemental OTA. Transistor sizing was performed according to the guidelines in [7] to get the desired transconductance values. The OTA is composed by a basic transconductor followed by a folded-cascode stage. In fact, the folded-cascode stage is used to provide impedance matching at every node and is shared (with proper scaling) by all the transconductors that are incident in the same node.

Monte Carlo electrical simulations were performed to estimate the possible variations in the transconductance step values of the time-variant transconductor. The obtained results show that worst cases will suffer deviations in the step levels below $5 \%$ of their intended value, which is below the $10 \%$ variation limit that was obtained in Step 1.

Concerning the linearity of the selected OTA, electrical simulation results give that coefficient $\alpha_{3}$ keeps below

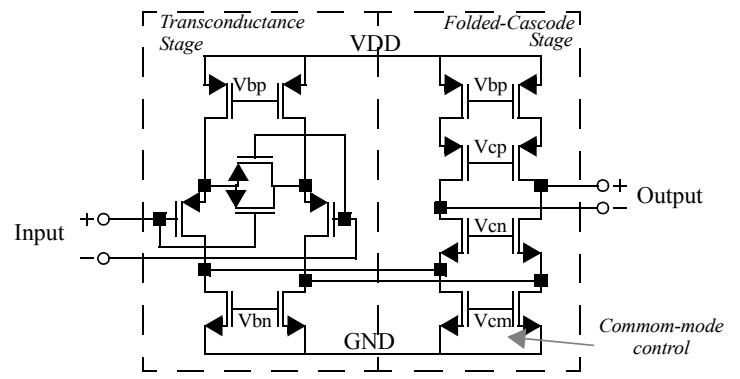

Fig. 9: Transistor level schematic of the implemented OTAs. 


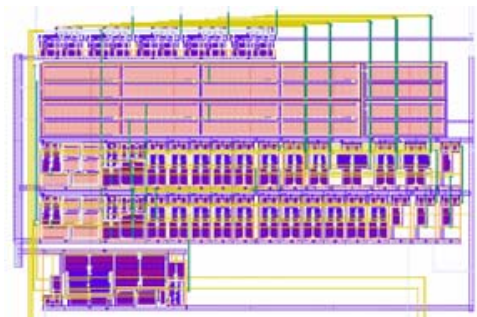

Fig. 10: Layout of the designed generator

$40 \mu \mathrm{V} / \mathrm{V}^{3}$ in the whole tuning range, which complies with the maximum tolerable value $\alpha_{3}=50 \mu \mathrm{V} / \mathrm{V}^{3}$ obtained in the previous step.

The modification of the input stage of the filter concludes the design of the generator.

\section{Simulation results}

The designed OTA-C generator was laid out following the recommended guidelines for mixed-signal circuits. Fig.10 shows the layout of the system. The core area is $395 \mu \mathrm{m}$ by $230 \mu \mathrm{m}$. Post-layout simulations of the extracted view were performed to check the performance of the generator. Fig.11 shows two output spectra. The frequency of the generated outputs have been set to $40.7 \mathrm{MHz}$, while their amplitudes have been varied from $175 \mathrm{mVpp}$ to $590 \mathrm{mVpp}$. In terms of spectral purity, the achieved THD and SFDR keep approximately between $70 \mathrm{~dB}$ for the $175 \mathrm{mVpp}$ signal, and $56 \mathrm{~dB}$ for the $590 \mathrm{mVpp}$ one. On the other hand, Fig. 12 shows the THD and SFDR figures obtained when sweeping the amplitude and the frequency of the generated output signal. Obtained results meet the goal specifications defined in Table I.

a)

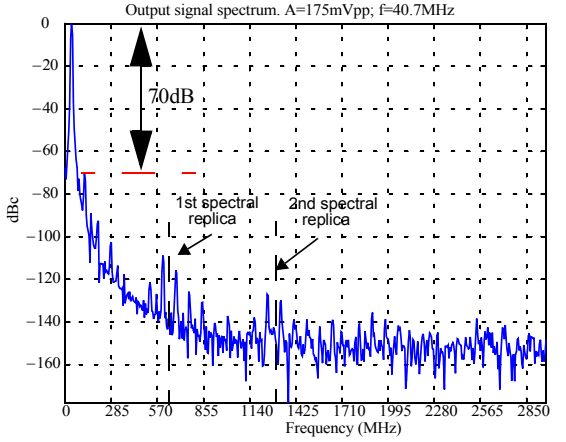

b)

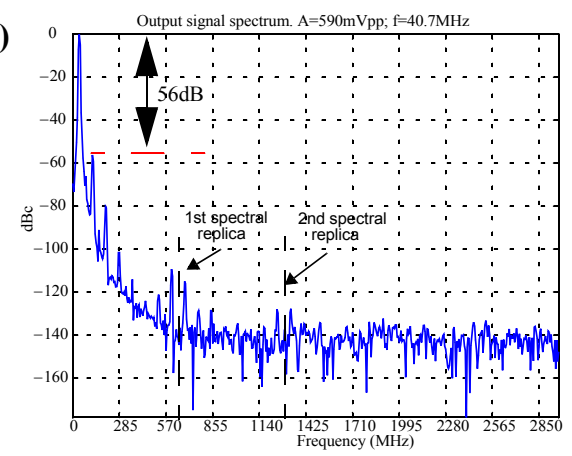

Fig. 11: Generator output spectra at $40.7 \mathrm{MHz}$ : a) $175 \mathrm{mVpp}$; b) $590 \mathrm{mVpp}$ a)

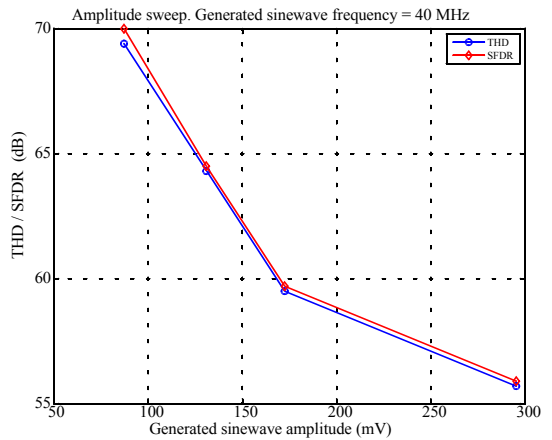

b)

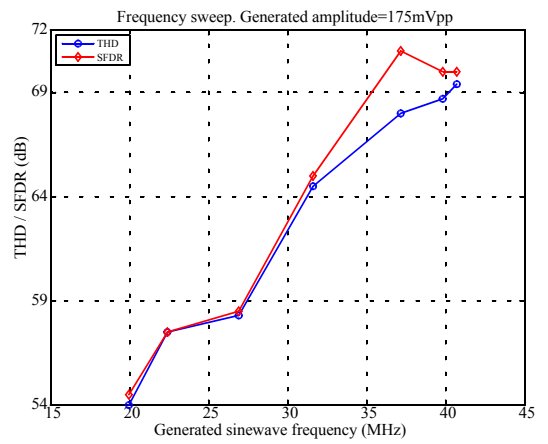

Fig. 12: a) Generator output THD and SFDR as a function of the output amplitude. b) Generator output THD and SFDR as a function of the output frequency

\section{CONCLUSIONS}

An efficient design methodology for the implementation of sinewave generators for analog and mixed-signal BIST applications have been presented. The given design guidelines have been validated providing a full design example. In particular, the design of a continuous-time OTA-C sinewave generator have been described, from the definition of the goal specifications to the post-layout simulations.

\section{ACKNOWLEDGEMENTS}

This work has been funded in part by the Spanish Government through a JAE-DOC contract, and projects TEST (TEC2007-68072/MIC), SR2 (TSI-020400-2008-71/MEDEA+ 2A105), TOETS (CATRENE CT302) and by the Junta de Andalucia through project ACATEX (P09-TIC-5386).

\section{REFERENCES}

[1] SIA Roadmap for semiconductors. http: //public.itrs.net

[2] B.Dufort and G.W.Roberts: "On-chip analog signal generation for mixed-signal Built-In-Self-Test". IEEE J. Solid-State Circuits, 1999, Vol 33, N 3, pp. 318-330

[3] A.K.Lu, et al: "A high quality analog oscillator using oversampling D/A conversion techniques". Trans. on Circ. and Sys. II', 1994, pp. 437-444.

[4] M.G.Méndez, et al: "An On-Chip Spectrum Analyzer for Analog Built-in Testing", J. of Electronic Testing, 2005, Vol 21, pp. 205-219.

[5] H.C.Patangia and B.Zenone:" A programmable switched-capacitor sinewave generator". Proc. Midwest Symp. on Circ. and Systems, 1994, pp. 165-168.

[6] M. J. Barragán, et al: "On-chip Analog Sinewave Generator with Reduced Circuitry Resources" Proc. of Midwest Symp. on Circ. and Systems, August 2006.

[7] J.E.Kardontchik: "Introduction to the design of OTA-C Filters". Ed. Kluwer Academic Publishers, 1992. 